\title{
Alta adesão aos medicamentos prescritos apesar de baixo comparecimento às reuniões de grupo entre pacientes do programa HIPERDIA
}

\author{
High adherence to prescribed medications despite low attendance at group meetings of patients \\ in the HIPERDIA program
}

\section{Alta adherencia a los medicamentos prescritos a pesar de baja asistencia a las reuniones de grupo entre los pacientes del programa HIPERDIA}

Rafaella Boszczovski. Faculdade Assis Gurgacz (FAG). Cascavel, PR, Brasil. rafaella_b@ hotmail.com

(Autora correspondente)

Dilson Fronza. Faculdade Assis Gurgacz (FAG). Cascavel, PR, Brasil. fronzad@ gmail.com

Mayara Angélica Bolson. Faculdade Assis Gurgacz (FAG). Cascavel, PR, Brasil. mayara_angelica@ hotmail.com

\section{Resumo}

Objetivo: O estudo teve por objetivo avaliar os índices de adesão às reuniões do grupo de hipertensos e diabéticos por meio do Sistema de Cadastramento e Acompanhamento de Hipertensos e Diabéticos (HIPERDIA), bem como o uso efetivo dos medicamentos da Unidade de Saúde da Família. Métodos: Durante o período de outubro de 2013 a março de 2014 foi realizado um estudo longitudinal e prospectivo, compreendendo uma coorte de pacientes de ambos os sexos, residentes na área de abrangência da Unidade de Saúde da Família São Francisco de Assis cadastrados no HIPERDIA. Os pacientes foram acompanhados durante as reuniões mensais nas comunidades, nas quais eram avaliadas as taxas de participação efetiva e a utilização das medicações por meio da checagem da saída mensal da medicação em estoque, vinculada ao paciente. Resultados: Dos 238 pacientes cadastrados no programa HIPERDIA, 226 deles $(94 \%)$ aderiram ao tratamento, e 12 pacientes (6\%) não utilizavam a medicação prescrita pelo médico da Unidade de Saúde. Apenas 34 pacientes (14,3\%) participaram das reuniões e retiravam a medicação nos encontros do HIPERDIA. Outros 192 pacientes $(80,7 \%)$ não participavam das reuniões e retiravam as medicações na Unidade de Saúde em algum momento durante o mês. Doze deles $(5 \%)$ não participaram das reuniões nem retiraram a medicação junto à Unidade de Saúde. Conclusão: A adesão ao tratamento do diabetes e da hipertensão arterial sistêmica foi considerada satisfatória, apesar do baixo índice de participação dos grupos nas reuniões do HIPERDIA.

\begin{abstract}
Objectives: The objectives of the study were to evaluate the rates of attendance at group meetings of patients with hypertension and diabetes mellitus, using the Registration and Monitoring of Hypertensive Diabetics System (HIPERDIA), and to evaluate the effective use of medications to treat these disorders, by means of monitoring patient collection of dispensed medication from the Family Health Unit. Methods: From October 2013 to March 2014, a prospective longitudinal study was conducted. The cohort comprised patients of both sexes, registered with HIPERDIA, and living in the area covered by the Family Health Unit São Francisco de Assis. Patients were followed up at monthly meetings in the community, where they were evaluated for effective participation rates and adherence to medications. The latter was done by reviewing the monthly dispensing of medication in stock, linked to individual patients. Results: Of the 238 patients enrolled in the HIPERDIA program, $226(94 \%)$ adhered to treatment, while $12(6 \%)$ did not. Only 34 patients (14.3\%) enrolled to attend, and to collect their medication at, the HIPERDIA group meetings. The other 192 patients $(80.7 \%)$ did not attend the meetings and collected their medications at the Health Unit at another time during the month. Twelve of these patients (5\%) did not participate in meetings or collect their medications at the Health Center. Conclusion: Adherence to treatment for diabetes and hypertension was satisfactory despite the low rate of participation in HIPERDIA group meetings.
\end{abstract}

Como citar: Boszczovski R, Fronza D, Bolson MA. Alta adesão aos medicamentos prescritos apesar de baixo comparecimento às reuniões de grupo entre pacientes do programa HIPERDIA. Rev Bras Med Fam Comunidade. 2016;11(38):1-7. http://dx.doi.org/10.5712/rbmfc11(38)984
Palavras-chave:

Hipertensão

Diabetes Mellitus

Terapêutica

Saúde da Família

Cooperação do Paciente
Keywords:

Hypertension

Diabetes Mellitus

Therapeutics

Family Health

Patient Adherence

Fonte de financiamento: declaram não haver.

Parecer CEP:

208/2013 (FAG), aprovado em

09/09/2013.

Conflito de interesses:

declaram não haver.

Procedência e revisão por pares: revisado por pares.

Recebido em: 30/07/2014.

Aprovado em: 22/01/2016. 


\section{Resumen}

Objetivo: El objetivo del estudio fue evaluar las tasas de adherencia a las reuniones del grupo hipertensos y diabéticos a través del sistema de registro y control de los hipertensos y diabéticos (HIPERDIA), así como estimar el uso efectivo de los medicamentos utilizados en el tratamiento de la Diabetes Mellitus e Hipertensión arterial sistémica. Métodos: De octubre de 2013 a marzo de 2014 se realizó un estudio prospectivo longitudinal, que comprende una cohorte de pacientes de ambos sexos, que viven en el área cubierta por la Unidad de Salud de la Familia São Francisco de Assis, inscritos en el HIPERDIA. Los pacientes fueron acompañados durante las reuniones mensuales en las comunidades, en las que se evaluaban las tasas de participación efectiva y la utilización de las medicaciones utilizadas por el paciente, a través Del control de la salida mensual de los medicamentos del stock. Resultados: De 238 pacientes inscritos en el programa HIPERDIA, 226 de ellos (94\%) adhirieron al tratamiento, y 12 pacientes (6\%) no utilizaron la medicación prescripta por el por el médico de la Unidade de Salud. Sólo 34 pacientes $(14,3 \%)$ participaron de las reuniones y recogieron la medicación em los encuentros HIPERDIA. Otros 192 pacientes $(80,7 \%)$ no participaron de las reuniones y recogieron los medicamentos en la Unidad de Salud en algún momento del mes. 12 de ellos (5\%) no participaron de las reuniones y tampoco recogieron los medicamento en la Unidad de Salud. Conclusión: La adherencia al tratamiento de la diabetes y de la hipertensión era satisfactoria a pesar del bajo índice de participación de los grupos en las reuniones HIPERDIA.

\section{Palabras clave:}

Hipertensión

Diabetes Mellitus

Terapéutica

Salud de la Familia

Cooperación del Paciente

\section{Introdução}

O diabetes mellitus (DM) e hipertensão arterial sistêmica (HAS) são condições clínicas frequentemente associadas e resultam em risco aumentado para complicações cardiovasculares. A HAS é uma condição clínica multifatorial caracterizada por níveis aumentados e sustentados da pressão arterial (PA), com valores $\geq 140 / 90 \mathrm{mmHg}$, e importante causa evitável de morbidade e mortalidade prematura. Representa, ainda, fator de risco significativo para acidente vascular cerebral isquêmico e hemorrágico, insuficiência cardíaca, infarto do miocárdio, doença renal crônica, alterações metabólicas e declínio cognitivo. ${ }^{1}$

O DM é um grupo heterogêneo de distúrbios metabólicos que apresenta em comum a hiperglicemia, a qual é o resultado de defeitos na ação da insulina, de sua secreção, ou ainda uma associação de ambos os mecanismos. É uma condição clínica crônica de alta morbimortalidade, culminando com perda importante na qualidade de vida, e uma das principais causas de mortalidade, insuficiência renal, amputação de membros inferiores, cegueira e doença cardiovascular, atingindo indivíduos em plena idade produtiva. ${ }^{2}$

A HAS e o DM possuem prevalência elevada e baixas taxas de controle, sendo consideradas como principais fatores de risco modificáveis e um dos maiores problemas de saúde pública. Em 2001, aproximadamente 7,6 milhões de mortes no mundo foram atribuídas à elevação da PA e ao DM, 54\% por acidente vascular encefálico (AVE) e $47 \%$ por doença isquêmica do coração (DIC). A maioria destes eventos é observada em países de baixo e médio desenvolvimento econômico e com maior prevalência em indivíduos com idades entre 45 e 69 anos. $^{3}$

Dentre as principais ações de promoção e prevenção de agravos na população em geral, está o diagnóstico precoce e tratamento da HAS e DM, e o seu acompanhamento por meio do Sistema de Cadastramento e Acompanhamento de Hipertensos e Diabéticos (HIPERDIA) do Governo Federal, que envolve reuniões dirigidas para pacientes hipertensos e diabéticos acompanhados em unidades de saúde, tendo como meta primordial a adesão do indivíduo ao tratamento proposto. Os mesmos recebem orientação sobre suas doenças e da importância do acompanhamento mensal, compartilham suas dificuldades e recebem os medicamentos necessários ao tratamento. Por meio do HIPERDIA, pode ser definido o perfil epidemiológico da população, e o consequente desencadeamento de estratégias de saúde pública, proporcionando a melhoria da qualidade de vida e redução do custo social. ${ }^{4}$ 
Abordagens bem sucedidas de atenção primária no acompanhamento dos pacientes hipertensos e diabéticos tendem a exigir múltiplas estratégias para alcançar altas taxas de adesão e controle do tratamento da HAS e DM. Para ser efetivo, um sistema deve ser simples e que permita à equipe multiprofissional de saúde identificar os fatores que dificultam a adesão, oferecer a facilidade para o acompanhamento e controle da pressão arterial, e motivar os pacientes para adoção de mudanças nos hábitos de vida. ${ }^{2}$

Pouco menos da metade das pessoas com HAS, no entanto, sabem que têm a doença. A maioria das pessoas cientes de que têm hipertensão recebem tratamento farmacológico, mas o controle clínico do tratamento ocorre apenas em uma parcela dos casos e a adesão tende a diminuir com o passar do tempo. ${ }^{5}$ Como consequência, a despeito de todo serviço assistencial oferecido pelo Sistema Único (SUS), cerca de $40 \%$ dos pacientes hipertensos não conseguem manter níveis de pressão arterial e glicemia controlados, devido à falta de adesão ao tratamento. ${ }^{6}$ Os motivos são muitas vezes justificáveis, tanto pela falta de informações com respeito ao caráter crônico da doença como também os relacionados aos efeitos colaterais observados em algumas das medicações utilizadas, prejudicando a aderência e manutenção ao tratamento. A falta de informações é especialmente observada quando os pacientes estão vinculados a serviços de saúde nos quais não se dispõe de um médico assistente ou profissional enfermeiro vinculado ao território do usuário. ${ }^{7}$

Melhorias neste cenário têm sido observadas com a introdução da estratégia do Programa Saúde da Família (PSF). O mesmo reorganiza a prática assistencial, possibilitando que não só o paciente, como também toda a família e comunidade, estejam vinculadas a um médico e a uma equipe de saúde específica. ${ }^{6}$ Em relação à atenção básica tradicional, o PSF possui um caráter substitutivo, atuando no território alvo por meio da realização do cadastramento domiciliar, diagnóstico situacional e ações dirigidas aos problemas de saúde juntamente com a comunidade. O programa busca o cuidado dos indivíduos e das famílias ao longo do tempo e tem por objetivo melhorar a saúde e a qualidade de vida, priorizando ações de prevenção e promoção da saúde de forma integral e contínua, tendo como foco a família e a comunidade. $^{8}$ Tais estratégias proporcionam cobertura mais ampla dos indivíduos suscetíveis, além de garantir maior eficiência, acesso e agilidade no acompanhamento dos pacientes hipertensos e diabéticos. ${ }^{9}$

Atingir alta cobertura da população definida como alvo é o componente mais importante no âmbito da atenção primária para que se obtenha significativa redução da morbimortalidade devido à HAS e DM. Entre as ações propostas pelo governo a fim de aumentar os índices de cobertura e o acompanhamento dos pacientes, estão o convite à participação das reuniões mensais do HIPERDIA. O sistema oferece o serviço de aferição da PA, glicemia capilar, marcação de consultas com o médico responsável se houver necessidade para adequação das medicações, orientações sobre o tratamento adequado, a visita das agentes comunitárias e o uso da mídia escrita e falada. ${ }^{10}$

O presente estudo se justificou pela contribuição na organização de um sistema para avaliar a adesão ao tratamento proposto de doenças prevalentes e de elevada morbimortalidade, contribuindo para o planejamento e ações em saúde coletiva.

\section{Métodos}

Estudo observacional e prospectivo conduzido para avaliar as taxas de adesão dos pacientes hipertensos e diabéticos ao programa HIPERDIA no período de outubro de 2013 a março de 2014. O trabalho ocorreu na área de abrangência da Unidade Saúde da Família São Francisco de Assis, zona 
rural de Cascavel, PR. A pesquisa foi apresentada ao Comitê de Ética em Pesquisa da Faculdade Assis Gurgacz e aprovada sob o parecer 280/2013.

Uma coorte compreendendo todos os 238 pacientes de ambos os sexos, cadastrados no grupo HIPERDIA e residentes na área de abrangência da Unidade de Saúde da Família São Francisco de Assis foi incluída no estudo. Os mesmos estavam subdivididos em uma das seis comunidades à que pertenciam, em ordem decrescente de distância da Unidade de Saúde: Colônia Sapucaia, Nova União, Alto Alegre, Aliança d'Oeste, Vargem Bonita e São Lucas.

Os pacientes com diagnóstico de DM e HAS eram convidados a participar das reuniões mensais referentes ao programa HIPERDIA organizadas pelas agentes comunitárias de saúde, independentemente da realização do estudo, visando o acompanhamento de suas doenças. Eram realizadas orientações higienodietéticas, aferição de pressão e outras medidas antropométricas (peso, circunferência abdominal e teste de glicemia capilar), bem como feita a entrega das medicações para o tratamento da HAS e do DM. Sendo assim, foi solicitada a autorização à Secretaria de Saúde do Município, juntamente com a Coordenadora responsável pela Unidade de Saúde, para acompanhar os grupos durante os encontros do HIPERDIA.

A partir dos achados de frequência nas reuniões, foram contabilizados quantos pacientes participaram e retiravam as medicações ao final do encontro, quantos deles não participaram mas aderiam ao tratamento e, por fim, quantos se recusavam ao tratamento farmacológico proposto pela equipe de saúde. A adesão foi aferida apenas por retirada do medicamento mensalmente na USF. Pacientes que relatassem não estar conseguindo manter os níveis pressóricos e de glicemia controlados, ou estivessem apresentando efeitos colaterais importantes com o uso das medicações, eram agendados para consulta com o médico da unidade e feito a readequação da prescrição.

De posse dos dados catalogados, foram avaliados os índices de adesão gerais para a população residente, e por comunidade assistida, bem como a periodicidade com que os pacientes retiravam as medicações fornecidas pela Unidade de Saúde. Os dados foram compilados e armazenados utilizando o software Excel 2010 e, posteriormente, analisados em um banco de dados informatizado.

\section{Resultados}

A população total residente e pertencente à área de abrangência para o período de outubro de 2013 a março de 2014 era constituída de 1417 usuários: 447 pacientes de zero a 17 anos e 970 adultos (18 anos ou mais); dentre os últimos, 238 estavam cadastrados no programa HIPERDIA. Os mesmos estavam distribuídos da seguinte forma: 185 eram somente hipertensos, 10 deles eram somente diabéticos e 43 apresentavam ambas as comorbidades.

A idade dos 238 pacientes cadastrados no programa para tratamento farmacológico variou de 28 a 91 anos ( $\bar{x}=58$ anos; $S D=12,02$ anos); desses, 226 aderiram ao tratamento, enquanto que outros 12 pacientes (todos hipertensos) não utilizavam qualquer das medicações instituídas pelo médico da USF.

A prevalência de hipertensão da população adulta observada na comunidade foi de $23,5 \%$ ( $n=228 / 970$ ), enquanto que a tratada foi de $22,27 \%$ ( $n=216 / 970)$, adesão de $94,7 \%(n=216 / 228)$ dos pacientes cadastrados 
no programa HIPERDIA. Do total dos 238 pacientes avaliados, apenas 34 (14,3\%) participavam das reuniões e retiravam a medicação nos encontros. A maioria, 80,7\% ( $n=192 / 238)$, dos pacientes não participava das reuniões, contudo, retirava as medicações em algum momento durante o mês na Unidade de Saúde. O controle da efetiva utilização das medicações era realizado por meio da checagem da saída mensal dos fármacos pelas saídas no estoque da medicação, vinculada ao paciente. A proporção dos participantes que utilizavam as medicações, aderindo ou não às reuniões, foi de $94,8 \%$ durante o período estudado. Os 12 pacientes remanescentes $(5,2 \%)$ não participaram das reuniões nem retiraram a medicação junto à Unidade de Saúde.

Quando observados os resultados relativos às subdivisões por microárea de abrangência, constatou-se grande disparidade de participação entre os grupos, tanto na presença nas reuniões quanto na adesão ao tratamento. Em relação aos demais, o grupo mais afastado da Unidade de Saúde, Colônia Sapucaia, apresentou maior participação proporcional tanto nas reuniões do HIPERDIA quanto na retirada das medicações na USF, e 11,8\% deles abandonaram o tratamento farmacológico. O grupo Nova União, segundo mais distante da Unidade, obteve $97,1 \%$ de adesão efetiva, e o segundo lugar em participação nas reuniões dos grupos de HIPERDIA. Apesar de nenhum participante ter estado presente no momento das reuniões no do grupo São Lucas, os mesmos obtiveram 100\% de adesão efetiva ao tratamento medicamento, pois todos eles retiraram a medicação em algum momento do respectivo mês. Os grupos do Alto Alegre, Aliança d'Oeste e Vargem Bonita obtiveram participações de 90,7\%, 95,8\% e 96,9\%, respectivamente.

\section{Discussão}

Apesar de toda a assistência prestada nos serviços de saúde, cerca de $40 \%$ dos pacientes hipertensos não conseguem controlar os níveis de pressão arterial e glicemia, devido à falta de adesão ao tratamento. Os motivos são muitas vezes justificáveis pelo déficit de informações fornecidas aos pacientes a respeito da doença e utilização das medicações, e seus possíveis efeitos colaterais. ${ }^{11}$ Esta falta de conhecimento ocorre quando os pacientes estão vinculados com os serviços de saúde e não a um médico ou profissional de saúde que permaneça trabalhando no local por dois anos ou mais no serviço. Entretanto, o tempo de permanência dos profissionais no mesmo serviço tem mudado com a implementação do PSF, possibilitando vínculo maior entre a equipe de saúde e o paciente. ${ }^{6}$

De acordo com dados da pesquisa conduzida pelo Ministério da Saúde (MS) Vigilância de Fatores de Risco e Proteção para Doenças Crônicas por Inquérito Telefônico - VIGITEL 2012, a média nacional de hipertensos é de $24,3 \%$. $^{12}$ O estudo obteve $23,5 \%$ de detecção do HAS, dados muito próximos aos estimados pelo Ministério da Saúde. A comunidade em questão possui pouco caráter migratório por se tratar de ambiente rural, e os pacientes efetivamente hipertensos são facilmente monitorados. O único centro local de saúde disponível aos mesmos é a USF São Francisco de Assis, e as medicações são todas retiradas na própria Unidade, ocasião em que os pacientes usualmente solicitam que sua pressão seja verificada.

Com relação ao DM, alguns estudos têm concluído que os pacientes portadores de DM tipo 2 não aderem ao tratamento proposto pela equipe de saúde no longo prazo, sendo que as taxas de não adesão variam de 40 a 90\%. ${ }^{13}$ Observamos discrepância dos dados encontrados na literatura em comparação 
com os resultados obtidos neste estudo. A taxa de DM tipo 2 diagnosticada foi de 3,7\%, e a taxa de DM tipo 2 tratada foi de $3,7 \%$, ou seja, $100 \%$ dos pacientes aderiram ao tratamento proposto pela equipe de saúde da USF São Francisco de Assis.

Com respeito à adesão efetiva ao tratamento da HAS e do DM, até o presente momento não existe consenso quanto às taxas de adesão consideradas aceitáveis. Entretanto, alguns estudos consideram razoáveis aderências acima de $80 \% .^{14}$ Pesquisas baseadas em amostras de pacientes atendidos em Unidades Básicas de Saúde (UBSs), que consideraram a procura por atendimento e tomadas diárias dos medicamentos, sugerem que pouco menos da metade desta população sabe que sofre da doença, e destes que recebem tratamento farmacológico, apenas $1 / 3$ têm controle pressórico adequado com a medicação. ${ }^{5} \mathrm{~A}$ adesão real à medicação anti-hipertensiva é estimada em torno de $50 \% .{ }^{15} \mathrm{O}$ estudo apresentou resultados muito distantes dos encontrados na literatura. Apesar de observada baixa participação nas reuniões dos grupos, no presente estudo a adesão foi de $94,8 \%$, bem acima dos patamares comumente observados em UBSs, demonstrando o impacto positivo do PSF no controle de doenças crônicas. ${ }^{15}$

O estudo certamente traz à discussão o real valor dos grupos de HIPERDIA como norteador do sucesso na aderência ao tratamento do DM e da HAS. As reuniões mensais tratando de assuntos relacionados à atenção preventiva parecem não ser o principal fator de adesão ao tratamento, demonstrado pelo desinteresse em participar das reuniões. Após a análise dos resultados que demonstraram baixa adesão às reuniões, considera-se que a boa relação familiar dos pacientes cadastrados no HIPERDIA com a equipe de saúde pode ter sido um fator importante envolvido na aderência ao tratamento.

Os principais motivos que justificaram a menor adesão das comunidades mais afastadas (Colônia Sapucaia e Nova União) foram atribuídos à distância para retirar a medicação na USF e a dificuldade de acesso devido às intempéries, como chuvas e consequentes problemas de transporte devido às estradas precárias. Os dois grupos que mais participavam das reuniões foram, possivelmente pela mesma razão, os mesmos com maiores falhas de adesão ao tratamento: os mais afastados da USF. A dificuldade de acesso à USF e a medicação oferecida ao final das reuniões do grupo do HIPERDIA tornava-se oportunidade única para adquirirem a medicação sem grandes deslocamentos, exceto quando necessitavam de renovação de receita ou readequação da prescrição, tornando-se necessário agendamento para consulta com o médico da USF, sendo essa uma oportunidade para retirar a medicação. Atribuímos esta sendo a razão da elevada participação nas reuniões mensais.

\section{Conclusões}

A adesão observada no estudo mostra o caráter factível da realização do controle das doenças crônicas como a HAS e o DM, independentemente das reuniões dos grupos de HIPERDIA. Taxas elevadas de adesão são possíveis ao se integrar a equipe de saúde no objetivo comum do controle pressórico e da glicemia. Os achados de $94,8 \%$ sugerem que o PSF é efetivo no controle dessas duas doenças crônicas de elevada morbimortalidade. A dificuldade em vincular a adesão ao tratamento com as reuniões mensais dos grupos de HIPERDIA demonstra, pelo menos na área de estudo em questão, que a frequência das reuniões poderia ser revista, sem prejudicar a aderência dos pacientes. 


\section{Referências}

1. National Institute for Health and Clinical Excellence. Hypertension: Clinical management of primary hypertension in adults. NICE clinical guideline 127. [Internet]. London; 011 [Cited 2016 Mar 4]. Available from: https://www.nice.org.uk/guidance/ cg127

2. Brasil. Ministério da Saúde, Secretaria de Atenção à Saúde, Departamento de Atenção Básica. Estratégias Para o Cuidado da Pessoa Com Doença Crônica. Diabetes Mellitus. Brasília: Ministério da Saúde; 2006.

3. Sociedade Brasileira de Cardiologia/Sociedade Brasileira de Hipertensão/Sociedade Brasileira de Nefrologia. VI Diretrizes Brasileiras de Hipertensão. Arq Bras Cardiol. 2010;95(1supl.1):1-51.

4. Brasil. Ministério da Saúde. DATASUS, Sistemas e Aplicativos, Epidemiológicos. HIPERDIA - Sistema de Cadastramento e Acompanhamento de Hipertensos e Diabéticos. [Internet]. Brasília: Ministério da Saúde; 2008 [Cited 2016 Mar 4]. Available from: http://hiperdia.datasus.gov.br/

5. Chow CK, Teo KK, Rangarajan S, Islam S, Gupta R, Avezum A, et al.; PURE (Prospective Urban Rural Epidemiology) Study investigators. Prevalence, awareness, treatment, and control of hypertension in rural and urban communities in high-, middle-, and low-income countries. JAMA. 2013;310(9):959-68. http://dx.doi.org/10.1001/jama.2013.184182 DOI: http:// dx.doi.org/10.1001/jama.2013.184182

6. Gomes TJO, Silva MVR, Santos AA. Controle da pressão arterial em pacientes atendidos pelo programa Hiperdia em uma Unidade de Saúde da Família. Rev Bras Hipertens. 2010;17(3):132-9.

7. Dias RS, Ávila Neto AG, Marques BJT, Machado PAS. Análise da prevalência de doenças crônicas e fatores de risco. Anais do Congresso Brasileiro de Medicina da Família e Comunidade; 2013 Maio 12. Belém, PA, Brasil.

8. Brasil. Ministério da Saúde. Secretaria de Atenção à Saúde, Departamento de Atenção Básica. Política Nacional de Atenção Básica/Ministério da Saúde, Secretaria de Atenção à Saúde, Departamento de Atenção Básica. 4a ed. Brasília: Ministério da Saúde; 2007.

9. Brasil. Ministério da Saúde. Secretaria de Atenção à Saúde. Política Nacional de Humanização. Cadernos HumanizaSUS. Brasília: Ministério da Saúde; 2010.

10. Carvalho Filha FSS, Nogueira LT, Viana LMM. Hiperdia: Adesão e percepção de usuários acompanhados pela estratégia saúde da família. Rev Rene. 2011;12(n.esp.):930-6.

11. Brasil. Ministério da Saúde. Secretaria de Atenção à Saúde. Departamento de Atenção Básica. Estratégias para o cuidado da pessoa com doença crônica: hipertensão arterial sistêmica/Ministério da Saúde, Secretaria de Atenção à Saúde, Departamento de Atenção Básica. Brasília: Ministério da Saúde; 2013.

12. Brasil. Ministério da Saúde. Secretaria de Vigilância em Saúde. Departamento de Vigilância de Doenças e Agravos não Transmissíveis e Promoção de Saúde. Vigitel Brasil 2012: vigilância de fatores de risco e proteção para doenças crônicas por inquérito telefônico. Ministério da Saúde, Secretaria de Vigilância em saúde, Departamento de Vigilância de Doenças e Agravos não Transmissíveis e Promoção da Saúde. Brasília: Ministério da Saúde; 2013.

13. Groff DP, Simões PWTA, Fagundes ALSC. Adesão ao tratamento dos pacientes diabéticos tipo Il usuários da estratégia saúde da família situada no bairro Metropol de Criciúma, SC. Arq Catarin Med. 2011;40(3):43-8.

14. Barbosa RGB, Lima NKC. Índices de adesão ao tratamento anti-hipertensivo no Brasil e no mundo. Rev Bras Hipertens. 2006;13(1):35-8.

15. Bastos-Barbosa RG, Ferriolli E, Moriguti JC, Nogueira CB, Nobre F, Ueta J, et al. Adesão ao tratamento e controle da pressão arterial em idosos com hipertensão. Arq Bras Cardiol. 2012;99(1):636-41. DOI: http://dx.doi.org/10.1590/S0066$782 X 2012005000054$ 